\title{
ON RESIDUE FORMULAS FOR CHARACTERISTIC NUMBERS
}

\author{
FRANCISCO GÓMEZ RUIZ \\ Departamento de Álgebra, Geometría y Topología, Universidad de Málaga \\ Ap. 59, 29080 Málaga, Spain \\ E-mail:fgomez@agt.cie.uma.es
}

\begin{abstract}
We show that coefficients of residue formulas for characteristic numbers associated to a smooth toral action on a manifold can be taken in a quotient field $\mathbf{Q}\left(X_{1}, \ldots, X_{r}\right)$. This yields canonical identities over the integers and, reducing modulo two, residue formulas for Stiefel Whitney numbers.
\end{abstract}

1. Introduction. The classical formulas of Baum and Cheeger, [2], and Bott, [5], give the Pontrjagin or Chern numbers as a sum of residues at the zeros of a Killing or holomorphic vector field. In this paper we substitute these residues by elements of the quotient field $\mathbf{Q}\left(X_{1}, \ldots, X_{r}\right)$ of the polynomial ring $\mathbf{Z}\left[X_{1}, \ldots, X_{r}\right], r$ being the dimension of the torus acting on the manifold $M$. My motivation for doing this work was actually trying to get rational numbers as residues. In case $M$ is a compact Riemannian manifold and $v$ is a Killing vector field on $M$, we substitute $v$ by the corresponding action of the associated torus $G$ : if $\varphi_{t}$ are the isometries of $M$ induced by $v$, then $G$ is the closure of the one parameter subgroup $\varphi_{t}$ in the compact group of all isometries of $M$. We observe that as $v$ changes in the Lie algebra of $G$ the Baum-Cheeger residues of $v$ factorize through a unique residue in $\mathbf{Q}\left(X_{1}, \ldots, X_{r}\right)$.

Residue formulas for Killing vector fields or toral actions and Pontrjagin classes have been given by N. Alamo and F. Gómez [1], P. Baum and J. Cheeger [2], R. Bott [5], F. Gómez [7], D. Lehmann [9]; for holomorphic vector fields and Chern classes by P. Baum and R. Bott [3], R. Bott [4]; for toral actions and Stiefel Whitney classes by J. Daccah and A. Wassermann [6].

We consider the following situation: $G$ is a torus of dimension $r$ acting smoothly on a compact connected oriented smooth manifold $M$ of dimension $2 m, F^{G}$ is the fixed point

2000 Mathematics Subject Classification: Primary 57R20, 57S15; Secondary 55R25.

Key words and phrases: toral action, characteristic class, fixed point set.

The paper is in final form and no version of it will be published elsewhere. 
set of the action of $G$ on $M$. By a theorem of Kobayashi [8], each connected component $F$ of $F^{G}$ is a submanifold.

We distinguish two cases:

(a) $\tau_{M}$ has a complex structure which is preserved by the action of $G$, and

(b) the dimension of $M$ is $4 k$.

In case $(\mathrm{a})$, suppose that $I=\left(i_{1}, \ldots, i_{m}\right)$ with $i_{1}+2 i_{2}+\cdots+m i_{m}=m$ and denote by $c_{I}(M)=\int_{M} c_{1}^{i_{1}} \ldots c_{m}^{i_{m}}$ the Chern number corresponding to $I$, where $c_{i}$ is the $i$ th Chern class of $\tau_{M}$.

In case $(\mathrm{b})$, suppose that $I=\left(i_{1}, \ldots, i_{k}\right)$ with $i_{1}+2 i_{2}+\cdots+k i_{k}=k$ and denote as usual by $p_{I}(M)=\int_{M} p_{1}^{i_{1}} \ldots p_{k}^{i_{k}}$ the Pontrjagin number corresponding to $I$, where $p_{i}$ is the $i$ th Pontrjagin class of $\tau_{M}$.

THEOREM. We associate canonically to the $G$-vector bundle $\tau_{M \mid F}$ a residue class $R e s_{I}(F)$ in the cohomology of $F$ with coefficients in the quotient field $\mathbf{Q}\left(X_{1}, \ldots, X_{r}\right)$ such that the Chern number $c_{I}(M)$, resp. the Pontrjagin number $p_{I}(M)$, is given by the sum $\sum_{F} \int_{F} \operatorname{Res}_{I}(F)$.

2. Residues. The canonical decomposition for the representations of $G$ on the tangent space of $M$ at the points of $F$ clearly induces a canonical $G$-vector bundle decomposition

$$
\tau_{M \mid F}=\nu_{0}^{F} \oplus \nu_{1}^{F} \oplus \cdots \oplus \nu_{s(F)}^{F}
$$

where $\nu_{0}^{F}=\tau_{F}$ is the tangent bundle to $F$ and $\nu^{F}=\nu_{1}^{F} \oplus \cdots \oplus \nu_{s(F)}^{F}$ is the normal bundle of the inclusion $F \hookrightarrow M$. It is well known that each $\nu_{j}^{F}$ admits a complex structure, unique up to conjugation, and corresponding integer vectors $n_{j}^{F} \in \mathbf{Z}^{r}, j=1, \ldots, s(F)$, so that the action of $G$ on $\tau_{M \mid F}$ is given by

$$
a .\left(v_{0} \oplus v_{1} \oplus \cdots \oplus v_{s(F)}\right)=v_{0} \oplus a^{n_{1}^{F}} \cdot v_{1} \oplus \cdots \oplus a^{n_{s(F)}^{F}} \cdot v_{s(F)},
$$

where, if $a=\left(a_{1}, \ldots, a_{r}\right) \in G$ and $n=\left(n_{1}, \ldots, n_{r}\right) \in \mathbf{Z}^{r}, a^{n}$ means $a_{1}^{n_{1}} \ldots a_{r}^{n_{r}} \in S^{1}$.

Set $X=\left(X_{1}, \ldots, X_{r}\right)$ and consider the linear polynomials

$$
\left\langle n_{j}^{F}, X\right\rangle=\sum_{k=1}^{r} n_{j k} X_{k} \in \mathbf{Z}\left[X_{1}, \ldots, X_{r}\right]
$$

$j=1, \ldots, s(F)$.

Set $\operatorname{rank}\left(\nu^{F}\right)=2 m^{F}, \operatorname{rank}\left(\nu_{j}^{F}\right)=2 m_{j}^{F}, j=1, \ldots, s(F)$.

In case $(\mathrm{a})$, suppose that $I=\left(i_{1}, \ldots, i_{m}\right)$ with $i_{1}+2 i_{2}+\cdots+m i_{m}=m$ and consider the symmetric polynomial in $m$ variables $c_{I}\left(Y_{1}, \ldots, Y_{m}\right)=\sigma_{1}\left(Y_{1}, \ldots, Y_{m}\right)^{i_{1}} \ldots$ $\sigma_{m}\left(Y_{1}, \ldots, Y_{m}\right)^{i_{m}}$.

Define the residue at $F$ associated to $I, \operatorname{Res}_{I}(F)$, by

$$
\frac{c_{I}\left(c_{01}^{F}+\left\langle n_{0}^{F}, X\right\rangle, \ldots, c_{0 m_{0}^{F}}^{F}+\left\langle n_{0}^{F}, X\right\rangle ; \ldots ; c_{s(F) 1}^{F}+\left\langle n_{s(F)}^{F}, X\right\rangle, \ldots, c_{s(F) m_{s(F)}^{F}}^{F}+\left\langle n_{s(F)}^{F}, X\right\rangle\right)}{\prod_{i=1}^{s(F)}\left(\prod_{j=1}^{m_{i}^{F}}\left(c_{i j}^{F}+\left\langle n_{i}^{F}, X\right\rangle\right)\right)},
$$

where $n_{0}^{F}=0$ and $c_{i 1}^{F}, \ldots, c_{i m_{i}^{F}}^{F}, i=0, \ldots, s(F)$, are formal variables of degree two so that $\sigma_{\lambda}\left(c_{i 1}^{F}, \ldots, c_{i m_{i}^{F}}^{F}\right)$ is the $\lambda$ th Chern class of $\nu_{i}^{F}$. 
Explicitly the numerator of $\operatorname{Res}_{I}(F)$ is given by

$$
\prod_{\lambda=1}^{m}\left(\sum_{\substack{\alpha_{0}+\cdots+\alpha_{s(F)}=\lambda \\
0 \leq \alpha_{i} \leq m_{i}}} c_{\alpha_{0}}(F) \prod_{i=1}^{s(F)}\left(\sum_{\alpha+\beta=\alpha_{i}}\left(\begin{array}{c}
m_{i}^{F}-\beta \\
\alpha
\end{array}\right)\left\langle n_{i}^{F}, X\right\rangle^{\alpha} c_{\beta}\left(\nu_{i}^{F}\right)\right)^{i_{\lambda}}\right)
$$

and the denominator by

$$
\prod_{i=1}^{s(F)} \sum_{\lambda+\mu=m_{i}^{F}}\left\langle n_{i}^{F}, X\right\rangle^{\lambda} c_{\mu}\left(\nu_{i}^{F}\right)
$$

where $c_{\mu}\left(\nu_{i}^{F}\right)$ is the $\mu$ th Chern class of $\nu_{i}^{F}$.

Observe that, since all the $n_{i}^{F}$ are nonzero, for $i \neq 0$, it makes sense to consider the inverse

$$
\frac{1}{\sum_{\lambda+\mu=m_{i}^{F}}\left\langle n_{i}^{F}, X\right\rangle^{\lambda} c_{\mu}\left(\nu_{i}^{F}\right)}=\frac{1}{\left\langle n_{i}^{F}, X\right\rangle^{m_{i}^{F}}} \frac{1}{1+\tilde{c}_{j}}, \quad i=1, \ldots, s(F)
$$

where

$$
\tilde{c}_{i}=1+\frac{c_{1}\left(\nu_{i}^{F}\right)}{\left\langle n_{i}^{F}, X\right\rangle}+\frac{c_{2}\left(\nu_{i}^{F}\right)}{\left\langle n_{i}^{F}, X\right\rangle^{2}}+\cdots+\frac{c_{m_{i}^{F}}\left(\nu_{i}^{F}\right)}{\left\langle n_{i}^{F}, X\right\rangle^{m_{i}^{F}}}
$$

and

$$
\frac{1}{1+\tilde{c}_{i}}=1-\tilde{c}_{i}+\tilde{c}_{i}^{2}-\tilde{c}_{i}^{3}+\cdots
$$

Therefore

$$
\begin{gathered}
\frac{1}{\prod_{i=1}^{s(F)}\left(\sum_{\lambda+\mu=m_{i}^{F}}\left\langle n_{i}^{F}, X\right\rangle^{\lambda} c_{\mu}\left(\nu_{i}^{F}\right)\right)} \\
=\frac{1}{\left\langle n_{1}^{F}, X\right\rangle^{m_{1}^{F}} \cdots\left\langle n_{s(F)}^{F}, X\right\rangle^{m_{t(F)}^{F}}} \frac{1}{\left(1+\tilde{c}_{1}\right) \cdots\left(1+\tilde{c}_{s(F)}\right)} .
\end{gathered}
$$

In case $(\mathrm{b})$, suppose that $I=\left(i_{1}, \ldots, i_{k}\right)$ with $i_{1}+2 i_{2}+\cdots+k i_{k}=k$ and consider the symmetric polynomial in $m$ variables $p_{I}\left(Y_{1}, \ldots, Y_{m}\right)=\sigma_{1}\left(Y_{1}^{2}, \ldots, Y_{m}^{2}\right)^{i_{1}} \ldots$ $\sigma_{k}\left(Y_{1}^{2}, \ldots, Y_{m}^{2}\right)^{i_{k}}$.

Define the residue at $F$ associated to $I, \operatorname{Res}_{I}(F)$, by

$$
\frac{p_{I}\left(c_{01}^{F}+\left\langle n_{0}^{F}, X\right\rangle, \ldots, c_{0 m_{0}^{F}}^{F}+\left\langle n_{0}^{F}, X\right\rangle ; \cdots ; c_{s(F) 1}^{F}+\left\langle n_{s(F)}^{F}, X\right\rangle, \ldots, c_{s(F) m_{s(F)}^{F}}^{F}+\left\langle n_{s(F)}^{F}, X\right\rangle\right)}{\prod_{i=1}^{s(F)}\left(\prod_{j=1}^{m_{i}^{F}}\left(c_{i j}^{F}+\left\langle n_{i}^{F}, X\right\rangle\right)\right)},
$$

where $n_{0}^{F}=0$ and $c_{i 1}^{F}, \ldots, c_{i m_{i}^{F}}^{F}, i=0, \ldots, s(F)$, are formal variables of degree two so that $\sigma_{\lambda}\left(\left(c_{01}^{F}\right)^{2}, \ldots,\left(c_{0 m_{0}^{F}}^{F}\right)^{2}\right)$ is the $\lambda$ th Pontrjagin class of $\nu_{0}^{F}$, and $\sigma_{\lambda}\left(c_{i 1}^{F}, \ldots, c_{i m_{i}^{F}}^{F}\right)$ is the $\lambda$ th Chern class of $\nu_{i}^{F}$, for $1 \leq i \leq s(F)$.

Explicitly the numerator of $\operatorname{Res}_{I}(F)$ is given by

$$
\prod_{\lambda=1}^{k}\left(\sum_{\substack{\alpha_{0}+\cdots+\alpha_{s(F)}=\lambda \\ 0 \leq \alpha_{i} \leq m_{i}}} p_{\alpha_{0}}(F) \prod_{i=1}^{s(F)} \tilde{\Phi}_{\alpha_{i}}\right)^{i_{\lambda}}
$$


with

$\tilde{\Phi}_{\alpha_{i}}=\Phi_{\alpha_{i}}\left(\sum_{\alpha+\beta=1}\left(\begin{array}{c}m_{i}^{F}-\beta \\ \alpha\end{array}\right)\left\langle n_{i}^{F}, X\right\rangle^{\alpha} c_{\beta}\left(\nu_{i}^{F}\right), \ldots, \sum_{\alpha+\beta=m_{i}^{F}}\left(\begin{array}{c}m_{i}^{F}-\beta \\ \alpha\end{array}\right)\left\langle n_{i}^{F}, X\right\rangle^{\alpha} c_{\beta}\left(\nu_{i}^{F}\right)\right)$

where $\Phi_{t}$ is given by the formula

$$
\sigma_{t}\left(Y_{1}^{2}, \ldots, Y_{m}^{2}\right)=\Phi_{t}\left(\sigma_{1}\left(Y_{1}, \ldots, Y_{m}\right), \ldots, \sigma_{m}\left(Y_{1}, \ldots, Y_{m}\right)\right) .
$$

The denominator is given, as in case (a), by

$$
\prod_{i=1}^{s(F)}\left(\sum_{\lambda+\mu=m_{i}^{F}}\left\langle n_{i}^{F}, X\right\rangle^{\lambda} c_{\mu}\left(\nu_{i}^{F}\right)\right)
$$

where $c_{\mu}\left(\nu_{i}^{F}\right)$ is the $\mu$ th Chern class of $\nu_{i}^{F}$.

Observe that actually, in both cases,

$$
\left\langle n_{1}^{F}, X\right\rangle^{2 m_{1}^{F}} \cdots\left\langle n_{s(F)}^{F}, X\right\rangle^{2 m_{s(F)}^{F}} \operatorname{Res}_{I}(F) \in \mathbf{Z}\left[X_{1}, \ldots, X_{r}\right] \otimes_{\mathbf{Z}} H^{*}(F ; \mathbf{Z}) .
$$

If $F$ is not reduced to a single point, endow $F$ with the orientation so that the given orientation on $\tau_{M \mid F}$ is the direct sum of the orientations on $\tau_{F}$ and $\nu^{F}$. It is obvious that $\int_{F} \operatorname{Re}_{I}(F) \in H_{*}\left(F ; \mathbf{Q}\left(X_{1}, \ldots, X_{r}\right)\right)$ is independent of the choice of the complex structure and corresponding orientation on $\nu^{F}$.

In case $F$ is one point, $\operatorname{Res}_{I}(F) \in \mathbf{Q}\left(X_{1}, \ldots, X_{r}\right)$ and we define then $\int_{F} \operatorname{Res} I(F)$ $=\epsilon_{F} \cdot \operatorname{Res}_{I}(F)$, where $\epsilon_{F}=1$ or -1 according to whether the complex orientation on $\nu^{F}=\tau_{M_{\left.\right|_{F}}}$ agrees or not with the given orientation on $\tau_{M_{\left.\right|_{F}}}$.

Again, $\int_{F} \operatorname{Res}_{I}(F)$ is independent of the choices.

To prove our theorem, replace the variables $X_{1}, \ldots, X_{r}$ by real numbers linearly independent over $\mathbf{Q}$, choose a $G$-invariant Riemannian metric on $M$ and consider the Killing vector field whose flow is given by $\varphi_{t}(x)=\left(e^{2 \pi t X_{1}}, \ldots, e^{2 \pi t X_{r}}\right) x$. Then, we follow the standard procedure of Bott, Baum, Cheeger of choosing $G$-invariant tubular neighbourhoods of $F$ and convenient Baum-Cheeger connections.

Corollary. If none of the integer vectors $n_{i}^{F}$ is of the form $2 \bar{n}_{i}^{F}$, with $\bar{n}_{i}^{F} \in \mathbf{Z}^{r}$, we derive from our main theorem a residue formula for the Stiefel Whitney numbers, by simply reducing modulo 2.

3. Examples and remarks. 1) As an illustration we consider the following action of the 2-dimensional torus $S^{1} \times S^{1}$ on $\mathbf{C} P^{2}$ :

$$
(a, b) \cdot\left\langle z_{0}, z_{1}, z_{2}\right\rangle=\left\langle z_{0}, a^{n_{11}} b^{n_{12}} z_{1}, a^{n_{21}} b^{n_{22}} z_{2}\right\rangle
$$

where we suppose $\left|n_{11} \cdot n_{22}-n_{12} \cdot n_{21}\right|=1$.

The three fixed points are $\langle 1,0,0\rangle,\langle 0,1,0\rangle$ and $\langle 0,0,1\rangle$.

The representation at $\langle 1,0,0\rangle$ is given by

$$
(a, b) \cdot\left\langle 1, \frac{z_{1}}{z_{0}}, \frac{z_{2}}{z_{0}}\right\rangle=\left\langle 1, a^{n_{11}} b^{n_{12}} \frac{z_{1}}{z_{0}}, a^{n_{21}} b^{n_{22}} \frac{z_{2}}{z_{0}}\right\rangle .
$$

The representation at $\langle 0,1,0\rangle$ is

$$
(a, b) \cdot\left\langle\frac{z_{0}}{z_{1}}, 1, \frac{z_{2}}{z_{1}}\right\rangle=\left\langle a^{-n_{11}} b^{-n_{12}} \frac{z_{0}}{z_{1}}, 1, a^{n_{21}-n_{11}} b^{n_{22}-n_{12}} \frac{z_{2}}{z_{1}}\right\rangle .
$$


The representation at $\langle 0,0,1\rangle$ is

$$
(a, b) \cdot\left\langle\frac{z_{0}}{z_{2}}, \frac{z_{1}}{z_{2}}, 1\right\rangle=\left\langle a^{-n_{21}} b^{-n_{22}} \frac{z_{0}}{z_{2}}, a^{n_{11}-n_{21}} b^{n_{12}-n_{22}} \frac{z_{1}}{z_{2}}, 1\right\rangle
$$

The Pontrjagin number $\sigma_{1}\left(\mathbf{C} P^{2}\right)$, which, of course, we know to be 3 , is given by the formula

$$
\begin{gathered}
\sigma_{1}\left(\mathbf{C} P^{2}\right)=\frac{\left(n_{11} X_{1}+n_{12} X_{2}\right)^{2}+\left(n_{21} X_{1}+n_{22} X_{2}\right)^{2}}{\left(n_{11} X_{1}+n_{12} X_{2}\right)\left(n_{21} X_{1}+n_{22} X_{2}\right)} \\
+\frac{\left(-n_{11} X_{1}-n_{12} X_{2}\right)^{2}+\left(\left(n_{21}-n_{11}\right) X_{1}+\left(n_{22}-n_{12}\right) X_{2}\right)^{2}}{\left(-n_{11} X_{1}-n_{12} X_{2}\right)\left(\left(n_{21}-n_{11}\right) X_{1}+\left(n_{22}-n_{12}\right) X_{2}\right)} \\
+\frac{\left(-n_{21} X_{1}-n_{22} X_{2}\right)^{2}+\left(\left(n_{11}-n_{21}\right) X_{1}+\left(n_{12}-n_{22}\right) X_{2}\right)^{2}}{\left(-n_{21} X_{1}-n_{22} X_{2}\right)\left(\left(n_{11}-n_{21}\right) X_{1}+\left(n_{12}-n_{22}\right) X_{2}\right)} .
\end{gathered}
$$

Set $\lambda=\frac{n_{11} X_{1}+n_{12} X_{2}}{n_{21} X_{1}+n_{22} X_{2}}$ and then

$$
\sigma_{1}\left(\mathbf{C} P^{2}\right)=\left(\lambda+\frac{1}{\lambda}\right)+\left(-\frac{\lambda}{1-\lambda}-\frac{1-\lambda}{\lambda}\right)+\left(1-\lambda+\frac{1}{1-\lambda}\right)=3 .
$$

2) Observe that, in example 1 , by giving real values to $X_{1}, X_{2}$ we cannot have that all three residues are integers, or equivalently, we cannot find a Killing vector field with integer residues.

3) The main theorem of this paper makes sense for $G$ being a finite abelian group; is it true in that case?

4) If we consider the Borel bundle $M_{G} \rightarrow B G$, with fibre $M$, associated to the universal bundle $E G \rightarrow B G$ and the $G$-manifold $M$; we can extend the action of $G$ on $M$ to an action of $G$ on $M_{G}$ in the obvious way and the fixed point set is then $B G \times F^{G}$ with $B G=\mathbf{C} P^{\infty} \times \stackrel{r}{ } \times \mathbf{C} P^{\infty}$. Therefore, the integral cohomology of $B G \times F^{G}$ is $H^{*}\left(F^{G}\right) \otimes \mathbf{Z}\left[X_{1}, \ldots, X_{r}\right]$ with degree of $X_{j}$ equal 2 . This explains why it is natural to consider rational residues in the variables $X_{1}, \ldots, X_{r}$.

Acknowledgments. This research was partially supported by a DGICYT grant (MTM2007-60016) and a Junta de Andalucía grant (FQM0213).

\section{References}

[1] N. Alamo and F. Gómez, Smooth toral actions on principal bundles and characteristic classes, Lecture Notes in Math. 1410, Springer, Basel, 1989, 1-26.

[2] P. Baum and J. Cheeger, Infinitesimal isometries and Pontrjagin numbers, Topology 8 (1969), 173-193.

[3] P. Baum and R. Bott, Singularities of holomorphic foliations, J. Diff. Geometry 7 (1972), 279-342.

[4] R. Bott, A residue formula for holomorphic vector fields, J. Diff. Geometry 1 (1967), 311-330.

[5] R. Bott, Vector fields and characteristic numbers, Mich. Math. J. 14 (1967), 231-244.

[6] J. Daccah and A. Wassermann, Stiefel-Whitney classes and toral actions, Topology Appl. 21 (1985), 19-26.

[7] F. Gómez, A residue formula for characteristic classes, Topology 21 (1982), 101-124. 
[8] S. Kobayashi, Fixed points of isometries, Nagoya Math. J. 13 (1958), 63-68.

[9] D. Lehmann, Résidus des sousvariétés invariantes d'un feuilletage singulier, Ann. Inst. Fourier 41 (1991), 211-258.

[10] J. W. Milnor and J. D. Stasheff, Characteristic Classes, Annals of Math. Studies 76, Princeton U. Press, 1972. 